\title{
A PROJECTED TRACE AND PARTWISE ISOMETRIC TRANSFORMATIONS OF ALMOST PERIODIC SYSTEMS
}

\author{
ROBERT E. VINOGRAD
}

\begin{abstract}
This paper is concerned with almost periodic linear systems of ODE's and their block diagonalization. The aim is to describe a class of all "best possible" (partwise isometric) block diagonalizing transformations and to construct two closely connected functions, a projected trace and its real part, the Liouville function, playing the same role for a subspace or a subbundle as the usual trace and its real part play in Liouville's formula. A theorem is proved which says that the projected trace of an almost periodic system is almost periodic. For the Liouville function of a one-dimensional spectral subbundle this result was obtained by Sacker and Sell. We extend it to the case of an invariant subbundle of higher dimension. If a system admits a Whitney sum invariant decomposition (e.g., a Sacker-Sell spectral decomposition) then partwise isometric block diagonalizing transformations are possible. This class includes the transformations of Bylov-Vinograd, Coppel, Palmer, and Ellis-Johnson. A theorem is proved which says that every partwise isometric transformation preserves Liouville functions of subbundles and turns them into real parts of usual block traces. In particular, for almost periodic systems, they remain almost periodic.
\end{abstract}

1. Introduction. To display the origin and use of projected traces, Liouville functions, and partwise isometric transformations, consider briefly the two following problems.

\subsection{Let a linear system}

$$
\dot{x}=A(t) x, \quad x \in X=R^{n} \text { or } C^{n},
$$

be given. For a subspace $V \subseteq X$ let $\hat{V}$ be the corresponding solution subspace: $\hat{V}=\{x(t): x(0) \in V\}$, and $V(t)$ denote the set of these solution values at $t$, i.e., $V(t)=\{x \in X: x=x(t), x(t) \in \hat{V}\}$. Choose a basis $x_{1}(t), \ldots, x_{m}(t)$ in $\hat{V}$ and let $G=G(t)=G_{x_{1} \cdots x_{m}}(t)$ be the Gram determinant: $G=\operatorname{det}\left|\left\langle x_{i}, x_{j}\right\rangle\right|$, where $\langle$,$\rangle is$ the scalar product in $X$. Define the Liouville function of $A(t)$ on $V(t)$ by

$$
L_{A, V}(t)=\frac{1}{2} \frac{d}{d t} \ln G(t)=\frac{\dot{G}}{2 G} .
$$

If $m=n$ so that $V=V(t)=X$, then $G=|W|^{2}$ where $W$ is the Wronskian, and by Liouville's formula $L_{A, X}(t)=\operatorname{Re} \operatorname{tr} A(t)$. An analogous formula for $m<n$ is to be found (3.5). It turns out that $L_{A, V}$ does not depend on the choice of the basis in $\hat{V}$.

Presented in October 1981 at the Tenth Annual Midwest Conference on Differential Equations at North Dakota State University and sponsored by NDSU; received by the editors November 30, 1981.

1980 Mathematics Subject Classification. Primary 34C35, 54H20, 58F10; Secondary 34K20.

Key words and phrases. Almost periodic, isometric transformations, Lyapunov transformations, ordinary differential equations, subbundles, trace.

${ }^{1}$ This research was supported by NSF grant MOS 8115323 . 
Moreover, for an ODE-flow and its invariant subbundle $(2.5,2.2), L_{A, V}$ is the real part of the projected trace, a continuous function on the base space $(4.1,4.2)$. In this case, if $A(t)$ is almost periodic, so is $L_{A, V}(t)$.

1.2 Suppose we want to find "the nicest simplification" of system (1). This means to transform (1) into a block diagonal system preserving the norms of solutions and the angles between them at least within each block. Below is the description of all such transformations.

Let $X=V_{1}+V_{2}$ be a direct sum with $\operatorname{dim} V_{k}=l_{k}, k=1,2$. Then $\hat{V}_{k}$ and $V_{k}(t)$, $k=1,2$, are defined and $X=V_{1}(t)+V_{2}(t)$ for every $t$.

A linear transformation $x=S(t) z$ (we always assume the existence of $S^{-1}$ and $\dot{S}$ ) takes (1) to a system

$$
\dot{Z}=B(t) Z, \quad B=S^{-1} A S-S^{-1} \dot{S} .
$$

This system is called: (i) block-diagonal corresponding to $\hat{V}_{k}\left(\mathrm{BD}-\hat{V}_{k}\right)$ if $B=$ $\operatorname{diag}\left\{B_{1}, B_{2}\right\}$ and to each $x(t) \in \hat{V}_{k}$ there corresponds a solution $z(t)$ of the $k$ th block of (3), $k=1,2$; (ii) $\hat{V}_{k}$-isometric or partwise isometric (to (1)) if $\langle x(t), \tilde{x}(t)\rangle=$ $\langle z(t), \tilde{z}(t)\rangle$ for $x(t), \tilde{x}(t) \in V_{k}, k=1,2$, and all $t$. So "BD- $\hat{V}_{k}$-isometric" denotes a system (3) satisfying both (i) and (ii). If (3) is $\hat{V}_{k}$-isometric to (1), then we will also refer to the transformation $S(t)$ as $\hat{V}_{k}$-isometric or partwise isometric.

The proof of the following proposition is left to the reader.

1.3 Proposition. System (3) is BD- $\hat{V}_{k}$ if and only if for every $t$ the first $l_{1}$ column vectors of the matrix $S(t)$ form a basis in $V_{1}(t)$ and its last $l_{2}$ columns do that in $V_{2}(t)$. Moreover, $S(t)$ is $\hat{V}_{k}$-isometric if and only if these bases are orthonormal.

1.4 Remarks. (a) It follows that $\|S(t)\| \leqslant 1$ for a partwise isometric $S(t)$ where $\|\cdot\|$ is Euclidean operator norm. (b) A $\hat{V}_{k}$-isometric $S(t)$ always exists; one of the simplest ways to get it is to pick solution bases in $\hat{V}_{k}, k=1,2$, apply the GramSchmidt orthonormalization process (separately in $V_{1}(t)$ and $V_{2}(t)$ ), and then take the obtained vectors as columns of $S(t)$; in addition, in this case if $A(t)$ is uniformly bounded, so is $\dot{S}(t)[2,4,5]$. (c) The same terminology and results are applicable (up to the obvious changes) to a direct decomposition $X=V_{1}+\cdots+V_{m}$ with $m>2$. (d) All transformations such as those by Bylov et al. [2], Coppel [3], Palmer [5], and Ellis and Johnson [4] are partwise isometric.

As an application of the Liouville function, it will be shown that any system (3) which is $\mathrm{BD}-\hat{V}_{k}$-isometric to (1) has $\operatorname{Re} \operatorname{tr} B_{k}(t)=L_{A, V_{k}}(t), k=1, \ldots, m$ (3.6). In addition, if (1) is almost periodic and the $V_{k}$ are subbundles, then the $L_{A, V_{k}}$ are almost periodic $(6.2,6.4)$.

2. Notations. We use the Sacker-Sell concept of a linear skew product flow restricted to the simplest case we need; see [6] for more details.

2.1 LSPF. Let $\pi$ be a flow on a product space $W=X \times Y$ where $Y$ is a connected compact metric space and $X=R^{n}$ or $C^{n}$. This means that $\pi$ is a continuous mapping from $W \times R$ to $W$ such that $\pi(w, 0)=w$ and $\pi(\pi(x, t), s)=\pi(x, t+s)$. The flow $\pi$ is a linear skew-product flow (LSPF) if $\pi$ has the form $\pi(x, y, t)=(\phi(x, y, t)$, $\sigma(y, t))$ where $\sigma(y, t)=y \cdot t$ is a flow on $Y$ and $\phi$ is linear in $x$. 
2.2 Subbundle. A subbundle $V$ in $X \times Y$ is a closed subset of $X \times Y$ such that for each $y \in Y$, (a) the fiber $V_{y} \equiv V(y)=\{x \in X:(x, y) \in V\}$ is a linear subspace of $X$ and (b) $\operatorname{dim} V_{y}=$ const. on $Y . V$ is $\pi$-invariant if it is an invariant set for the flow $\pi$. If $V$ is a subbundle then (i) there is a (uniquely determined) hermitian projection $P_{y}: X \rightarrow X$ with range $V_{y}$ where $P_{y}$ is continuous in $y$ on $Y$; (ii) for each $y_{0} \in Y$ there is a neighborhood $U$ of $y_{0}$ in $Y$ such that a basis $x_{1}(y), \ldots, x_{m}(y)$ in $V_{y}=V(y)$ can be chosen to be continuous in $U$.

2.3 Whitney sum. The notation $X \times Y=V_{1}+\cdots+V_{m}$ (Whitney sum) means that the $V_{k}$ are subbundles such that $X=V_{1}(y)+\cdots+V_{m}(y)$ is a direct sum for every $y \in Y$. A Whitney sum is $\pi$-invariant if the $V_{k}$ are $\pi$-invariant, $k=1, \ldots, m$.

2.4 Almost periodic flow. A flow $\sigma(y, t)=y \cdot t$ on $Y$ is minimal if every orbit $\{y \cdot t: t \in R\}$ is dense in $Y$. A flow $\sigma$ is almost periodic if given $\varepsilon>0$ there is $\delta>0$ such that $d(y, z)<\delta$ implies $d(y \cdot t, z \cdot t)<\varepsilon$ for all $t \in R$. If $\sigma$ is almost periodic and $f: Y \rightarrow C^{k}$ is continuous then for each $y \in Y$ the function $t \rightarrow f(y \cdot t)$ is Bohr almost periodic.

2.5ODE-flow. There is a way to generate an LSPF $\pi$ on $X \times Y$ by starting with a flow $\sigma(y, t)=y \cdot t$ on $Y$. Let $L(X)$ be the set of all linear mappings (operators) $X \rightarrow X$ (by using a standard basis in $X$ one can also view $L(X)$ as the set of all ( $n \times n)$-matrices). Now let $A: Y \rightarrow L(X)$ defined by $y \rightarrow A_{y}$ be continuous. Then for each $y \in Y$ the mapping $t \rightarrow A_{y}(t) \equiv A_{y \cdot t}$ represents a continuous operatorvalued (matrix-valued) function of $t$. Let $\phi(x, y, t)$ denote the solution of the initial value problem

$$
\dot{x}=A_{y}(t) x, \quad x(0)=x .
$$

Then $\pi(x, y, t)=(\phi(x, y, t), y \cdot t)$ is an LSPF on $X \times Y$. We call it the ODE-flow described by $\left(4_{y}\right)$ or simply the ODE-flow of $\left(4_{y}\right)$. Note that if $\sigma$ is almost periodic, so is every system $\left(4_{y}\right)$ (i.e. its matrix $A_{y}(t)$ ). An individual almost periodic system (1) can be always included in a minimal almost periodic ODE-flow $\left(4_{y}\right)$ [6].

2.6 LP-transformations. A linear transformation $x=S(t) z$ is said to be Lyapunov-Perron (LP) if $S(t), S^{-1}(t)$, and $\dot{S}(t)$ are (uniformly) bounded. If $S(t)$ is bounded, then $S^{-1}(t)$ is bounded if and only if $\inf _{t}|\operatorname{det} S(t)|>0$.

3. The projected trace and the Liouville function. Let $X=R^{n}$ or $C^{n},\langle$,$\rangle denote$ scalar product, $A: X \rightarrow X$ be linear, and $V$ be a linear subspace of $X$. Then the hermitian projection $P: X \rightarrow X, P^{*}=P$, with range $P=V$ is uniquely determined and $(P A) V \subseteq V$. Therefore the restriction $(P A)^{\mathcal{V}}=P A \mid V: V \rightarrow V$ makes sense and its $\operatorname{trace} \operatorname{tr}(P A)^{0}$ is defined.

3.1 Definition. The projected trace of $A$ on $V$ is given by $\operatorname{Tr}(A, V)=\operatorname{tr}(P A)^{v}$.

3.2 REMARK. It is easily seen that $\operatorname{tr}\left(A+A^{*}, V\right)=\operatorname{Re} \operatorname{Tr}(A, V)$.

Now choose a basis $x_{1}, \ldots, x_{m}$ in $V$, put $G=\operatorname{det}\left|\left\langle x_{i}, x_{j}\right\rangle\right|$ (the Gram determinant) and let $G_{r}(A)$ denote the determinant obtained from $G$ by replacing its $r$ th row $\left\langle x_{r}, x_{1}\right\rangle \cdots\left\langle x_{r}, x_{m}\right\rangle$ by the row $\left\langle A x_{r}, x_{1}\right\rangle \cdots\left\langle A x_{r}, x_{m}\right\rangle$.

3.3 Lemma. $\sum_{r=1}^{m} G_{r}(A) / G=\operatorname{Tr}(A, V)$ (so the left-hand side does not depend on the choice of the basis in $V$ ). 
Proof. Since $(P A) V \subseteq V$ and $x_{1}, \ldots, x_{m}$ is a basis in $V$, one has

$$
P A x_{k}=(P A)^{v} x_{k}=\sum_{i=1}^{m} \alpha_{i k} x_{i}, \quad k=1, \ldots, m .
$$

Next, $\left\langle A x_{r}, x_{j}\right\rangle=\left\langle A x_{r}, P x_{j}\right\rangle=\left\langle P A x_{r}, x_{j}\right\rangle$ because $P x_{j}=x_{j}$ and $P^{*}=P$. Now the entries of the $r$ th row in $G_{r}(A)$ become

$$
\left\langle A x_{r}, x_{j}\right\rangle=\left\langle P A x_{r}, x_{j}\right\rangle=\sum_{i=1}^{m} \alpha_{i r}\left\langle x_{i}, x_{j}\right\rangle
$$

so that $G_{r}(A)$ can be expanded into $\sum_{i} \alpha_{i r} G_{r i}$ where $G_{r r}=G$ and all the remaining determinants $G_{r i}=0$ because their $r$ th and $i$ th rows are equal. So $G_{r}(A)=\alpha_{r r} G$ and 3.3 follows since $\sum_{r} \alpha_{r r}=\operatorname{tr}(P A)^{v}=\operatorname{Tr}(A, V)$.

3.4 ReMARK. By 3.2, $\sum_{r=1}^{m} G_{r}\left(A+A^{*}\right) / G=2 \operatorname{Re} \operatorname{Tr}(A, V)$.

3.5 LEMMA. The Liouville function $L_{A, V}(t)(2)$ does not depend on the choice of the basis in $\hat{V}$ and equals $\operatorname{Re} \operatorname{Tr}(A(t), V(t))$.

Proof. Look at (2) and differentiate $G(t)$ by rows. Since the $x_{k}$ are solutions to (1), we have

$$
\frac{d}{d t}\left\langle x_{r}, x_{j}\right\rangle=\left\langle A x_{r}, x_{j}\right\rangle+\left\langle x_{r}, A x_{j}\right\rangle=\left\langle\left(A+A^{*}\right) x_{r}, x_{j}\right\rangle .
$$

Therefore $\dot{G}=\Sigma_{r} G_{r}\left(A+A^{*}\right)$. Now 3.5 follows from (2) and 3.4.

3.6 THEOREM (SEE 1.2 AND 1.4(c)). Let a system (1) and a direct decomposition $X=V_{1}+\cdots+V_{m}$ be given. If a system (3) is BD- $\hat{V}_{k}$-isometric to (1), then for each block of $B=\operatorname{diag}\left\{B_{1}, \ldots, B_{m}\right\}$, one has

$$
\operatorname{Re} \operatorname{tr} B_{k}(t)=\operatorname{Re} \operatorname{Tr}\left(A(t), V_{k}(t)\right), \quad k=1, \ldots, m .
$$

In particular, all such systems (3) have the same $\operatorname{Re} \operatorname{tr} B_{k}(t), k=1, \ldots, m$.

Proof. By hypothesis, (3) is the result of a $\hat{V}_{k}$-isometric transformation $x=S(t) z$ of (1). Since $S(t)$ preserves scalar products within each subspace $V_{k}(t)$, it also preserves Gram determinants: $G_{x_{1} \cdots x_{m}}(t)=G_{z_{1} \cdots z_{m}}(t)$. Hence the Liouville functions of both sides are also equal. But the first of them is $\operatorname{Re} \operatorname{Tr}\left(A, V_{k}\right)$ by 3.5 and the second is $\operatorname{Re} \operatorname{tr} B_{k}$ by Liouville's formula (cf. 1.1).

3.7 REMARK. So far $S(t)$ need not be Lyapunov-Perron. The condition of partwise isometry implies only $\|S(t)\| \leqslant 1$ and the existence (but not boundedness) of $S^{-1}(t)$; it also says nothing about $\dot{S}(t)$. In $\$ 5 S(t)$ becomes LP.

4. The projected trace on a subbundle. Let $V$ be a subbundle of $X \times Y$ so that for each $y \in Y$ the fiber $V_{y}$ is a linear subspace of $X$. Next let a mapping $A: Y \rightarrow L(X)$ : $y \rightarrow A_{y}$ be given so that $A_{y}: X \rightarrow X$ is linear. Then the projected trace $\operatorname{Tr}\left(A_{y}, V_{y}\right)$ is defined for every $y \in Y$.

4.1 Definition. The projected trace of $A$ on $V$ is the function $\operatorname{Tr}[A, V]: Y \rightarrow C$ given by $\operatorname{Tr}[A, V](y)=\operatorname{Tr}\left(A_{y}, V_{y}\right)$.

4.2 LeMMA. If $A$ is continuous on $Y$, so is $\operatorname{Tr}[A, V]$. 
Proof. Pick $y_{0} \in Y$. In a neighborhood $U$ of $y_{0}$ in $Y$ one can choose a basis in $V_{y}$ depending continuously on $y$ in $U$. Then its Gram determinant $G$ as well as $G_{r}(A)$ are continuous in $U$. By 3.3 so is $\operatorname{Tr}\left(A_{y}, V_{y}\right)=\operatorname{Tr}[A, V](y)$. In particular $\operatorname{Tr}[A, V]$ is continuous at $y_{0}$. Since $y_{0} \in Y$ is arbitrary, 4.2 is proved.

5. ODE-flows with invariant Whitney sums. Let an ODE-flow $\pi$ of $\left(4_{y}\right)$ admit a $\pi$-invariant Whitney sum decomposition $X \times Y=V_{1}+\cdots+V_{m}$ (e.g., it may be the Sacker-Sell spectral decomposition). Recall our "double" notation $V_{y}=V(y)$ for a fiber. For a fixed $y \in Y$ one can view $V_{k}(y)$ as an initial value subspace of $X$ for the system $\left(4_{y}\right)$, so that the notations from 1.1 are applicable (such as $\hat{V}_{k}(y)$, a solution subspace, or $V_{k}(y)(t)$, the set of corresponding solution values at $\left.t\right)$.

5.1 REMARK. In fact this time $V_{k}(y)(t)=V_{k}(y \cdot t)$ because $V_{k}$ is invariant for $\pi=(\phi, y \cdot t)$ and $\phi$ just denotes solutions.

In the following theorem we shorten " $\hat{V}_{k}(y)$-isometric for a fixed $y$ " to " $V_{k}$ isometric".

5.2 TheOREM. Let $X \times Y=V_{1}+\cdots+V_{m}$ be a $\pi$-invariant Whitney sum decomposition for the ODE-flow of $\left(4_{y}\right)$. Then for each $y \in Y$ any $V_{k}$-isometric transformation $S(t)$ of the system $\left(4_{y}\right)$ is Lyapunov-Perron provided $\dot{S}(t)$ is uniformly bounded.

Proof. By 1.4(a) and 2.6 the only question is whether $\inf _{t}|\operatorname{det} S(t)|>0$. Suppose that det $S\left(t_{j}\right) \rightarrow 0$ for a sequence $\left\{t_{j}\right\}$. We will denote subsequences again by $\left\{t_{j}\right\}$ if necessary. Then $y \cdot t_{j} \rightarrow y_{0}$ since $Y$ is compact. Let $F_{k}(t)$ be the orthonormal frame consisting of the columns of $S(t)$ which span $V_{k}(y \cdot t)(1.3,1.4(\mathrm{c}))$. Then $F_{k}\left(t_{j}\right) \rightarrow F_{k}^{0}$, an orthonormal frame in $V_{k}\left(y_{0}\right) . F_{k}^{0}$ spans $V_{k}\left(y_{0}\right)$ because $\operatorname{dim} V_{k}(y)=$ const. Hence the collection of vectors $\left\{f_{l}\right\}=\cup_{k} F_{k}^{0}$ spans the whole space $X=V_{1}\left(y_{0}\right)$ $+\cdots+V_{m}\left(y_{0}\right)$. This implies $D_{0} \neq 0$ where $D_{0}$ is the determinant of the matrix with columns $\left\{f_{l}\right\}$. But $D_{0}=\lim S\left(t_{j}\right)=0$, a contradiction

5.3 REMARK. The requirement " $\dot{S}$ bounded" cannot be omitted; e.g., if $m=1$, i.e., $X \times Y=V_{1}$, then any unitary $S(t)$ is $V_{1}$-isometric and $\|S(t)\|=\left\|S^{-1}(t)\right\|=1$, but $\|\dot{S}(t)\|$ can be made arbitrary; the same thing holds for $m>1$. However a $V_{k}$ isometric $S(t)$ with $\dot{S}(t)$ bounded always exists (1.4(b)). Hence by 5.2 a LyapunovPerron $V_{k}$-isometric $S(t)$ exists too.

\section{Almost periodic ODE-flow.}

6.1 THEOREM. Let an ODE-flow $\pi$ on $X \times Y$ described by $\left(4_{y}\right)$ be almost periodic. If $V$ is a $\pi$-invariant subbundle in $X \times Y$, then for every $y \in Y$ the projected trace of $A_{y}(t)$ on $V_{y}(t)$ is Bohr almost periodic. More explicitly,

$$
\operatorname{Tr}\left(A_{y}(t), V_{y}(t)\right)=\operatorname{Tr}[A, V](y \cdot t)
$$

where $\operatorname{Tr}[A V]: Y \rightarrow C$ is continuous.

Proof. $A_{y}(t)=A_{y \cdot t}$ by the very notation (2.5), and $V_{y}(t)=V_{y \cdot t}$ by 5.1. Now (5) is merely 4.1. $\operatorname{Tr}[A, V]$ is continuous on $Y$ by 4.2 because $A$ is continuous by the construction (2.5). Therefore $\operatorname{Tr}[A, V](y \cdot t)$ is Bohr almost periodic (2.4). 
6.2 Corollary. The Liouville function of $A_{y}(t)$ on $V_{y}(t)$ is also almost periodic and $L_{A_{y}, V_{y}}(t)=\operatorname{Re} \operatorname{Tr}[A, V](y \cdot t)$. This follows from 6.1 and 3.5.

6.3 REMARK. For a one-dimensional spectral subbundle this result was proved by Sacker and Sell [7, p. 351, proof of Theorem 7]. (If $\operatorname{dim} V=1$ then the Liouville function is $(d / d t)\|\phi(t)\|$ where $\phi$ is a solution of $\left(4_{y}\right)$ with $\phi(0) \in V_{y}$.) See also [1] for the multidimensional case in terms of exponential separation.

6.4 THEOREM. Let an ODE-flow $\pi$ of $\left(4_{y}\right)$ be almost periodic and admit a $\pi$-invariant Whitney sum decomposition $X \times Y=V_{1}+\cdots+V_{m}$. Then for each $y \in Y$ : (I) any $V_{k}$-isometric transformation $x=S(t) z$ with $\dot{S}(t)$ uniformly bounded is LyapunovPerron; (II) such transformations exist; (III) all resulting block diagonal systems (3) (BD- $V_{k}$-isometric) have the same real parts of block traces, namely

$$
\operatorname{Re} \operatorname{tr} B_{k}(t)=\operatorname{Re} \operatorname{Tr}\left[A, V_{k}\right](y \cdot t), \quad k=1, \ldots, m,
$$

which are Bohr almost periodic.

The proof follows directly from 5.2, 5.3, 3.6, and 6.1.

REMARK. The theorem is applicable to all transformations listed in 1.4(d). In particular, it is valid for Palmer's [5] and Ellis and Johnson's [4] transformations $S$ which are constructed not only for individually fixed $y \in Y$ but are also defined and continuous on a flow-homomorphic extension of $Y$.

\section{REFERENCES}

1. B. F. Bylov and R. E. Vinograd, Separation and E-splitting of almost periodic systems, Differential Equations 13 (1977), 947-953. (Russian)

2. B. F. Bylov, R. E. Vinograd, D. M. Grobman and V. V. Nemytskii, Theory of Lyapunov exponents and its application to problems of stability, Nauka, Moscow, 1966. (Russian)

3. W. A. Coppel, Dichotomies in stability theory, Lecture Notes in Math., vol. 629, Springer-Verlag, Berlin, 1978.

4. R. Ellis and R. Johnson, Topological dynamics and linear differential systems, J. Differential Equations (to appear).

5. K. J. Palmer, On the reducibility of almost periodic systems of linear differential equations, J. Differential Equations 36 (1980), 374-390.

6. R. J. Sacker and G. R. Sell, Existence of dichotomies and invariant splittings for linear differential systems. I, J. Differential Equations 15 (1974), 429-458.

7. __ A spectral theory for linear differential systems, J. Differential Equations 27 (1978), 320-358.

Department of Mathematics, University of Southern California, los Angeles, California 90007

Department of Mathematical Sciences, North Dakota State University, Fargo, North DAKOTA 58105 (Current address) 